\title{
Virulence gene profiling of enterohemorrhagic (EHEC) and enteropathogenic (EPEC) Escherichia coli strains: a basis for molecular risk assessment of typical and atypical EPEC strains
}

Marie Bugarel ${ }^{1}$, Annett Martin $^{2}$, Patrick Fach ${ }^{1}$ and Lothar Beutin ${ }^{3 *}$

\begin{abstract}
Background: Enterohaemorrhagic E. coli (EHEC) can cause severe disease such as bloody diarrhoea and haemolytic uraemic syndrome in humans. Besides production of Shiga toxins, the presence of LEE (eae-gene) and non-LEE (nle) encoded effector genes harboured on O-islands OI-122, $\mathrm{Ol-71}$ and $\mathrm{OI}-57$ is associated with EHEC virulence and their frequency in outbreaks. Genes encoded by the EHEC-plasmid are putative virulence markers of EHEC. EHECplasmids, LEE and non-LEE effector genes have also been detected in some strains of enteropathogenic E. coli (EPEC). The objective of this study was to analyze the relationship between EHEC and EPEC for virulence genes encoded by genomic O-islands and by the EHEC-plasmids.

Results: Nle genes ent/espL2, nleB and nleE (OI-122), nleA, nleF and nleH1-2 (OI-71), nleG5-2 and nleG6-2 (OI-57), espK (CP-933N) and the EHEC-plasmid encoded genes ehxA, espP, etpD and katP were searched in 73 typical and in 235 atypical enteropathogenic E. coli (EPEC) strains. Typical and atypical EPEC each fall into two clusters. Cluster 1 typical $(n=46)$ and atypical $(n=129)$ EPEC strains were characterized by the presence of Ol-122 encoded genes and grouped together with 64 investigated EHEC strains. Cluster 2 typical $(n=27)$ and atypical $(n=106)$ strains grouped together with 52 LEE-negative, Shiga toxin-producing E. coli (STEC) and with 21 apathogenic E. coli strains. Typical EPEC Cluster 1 strains belonged to serotypes frequently involved in severe illness and outbreaks in children (O111:H2, O114:H2, O55:H6, O127:H6 and O142:H6). Atypical EPEC Cluster 1 strains were characterized by serotypes related to EHEC (O26:H11, O55:H7, O145:H28, O103:H2 and O103:H25).

Conclusion: The Ol-122 encoded nleB gene was found to be most closely associated with Cluster 1 strains and may serve as a diagnostic tool for the identification of virulent EHEC and EPEC seropathotypes. OI-71 encoded genes nleA, nleF and nleH1-2 are less associated with Cluster 1 strains. EHEC-plasmid, Ol-57 and CP-933 associated genes showed only weak similarities with virulent Cluster 1 EHEC and EPEC strains.
\end{abstract}

\section{Background}

Escherichia coli strains that cause diarrhoea in humans have been divided into different pathotypes according to their virulence attributes and the mechanisms involved in the disease process $[1,2]$. Five major groups of intestinal pathogenic strains have been established, such as enteropathogenic E. coli (EPEC), enterohemorrhagic E. coli

\footnotetext{
* Correspondence: Lothar.Beutin@bfr.bund.de

${ }^{3}$ National Reference Laboratory for Escherichia coli (NRL-E.coli), Federal Institute for Risk Assessment (BfR), Diedersdorfer Weg 1 D-12277 Berlin, Germany

Full list of author information is available at the end of the article
}

(EHEC), enteroaggregative E. coli (EAEC), enterotoxigenic E. coli (ETEC) and enteroinvasive E. coli (EIEC).

While EPEC is a major cause of infantile diarrhoea in the developing world, EHEC is associated with foodborne outbreaks in the developed world and can cause bloody diarrhoea, haemorrhagic colitis ( $\mathrm{HC}$ ) and the Haemolytic Uraemic Syndrome (HUS) due to the elaboration of Shiga toxin (Stx). More than 400 E. coli serotypes that produce Shiga toxins (STEC) have been described [3]. A small number of these have been shown to be implicated in severe disease such as HC and HUS in humans. A classification scheme has been
C Biomed Central

C 2011 Bugarel et al; licensee BioMed Central Ltd. This is an Open Access article distributed under the terms of the Creative Commons Attribution License (http://creativecommons.org/licenses/by/2.0), which permits unrestricted use, distribution, and reproduction in any medium, provided the original work is properly cited. 
established to group STEC strains into the five seropathotype groups A-E depending on the severity of disease, the incidence of human infections and the frequency of their involvement in outbreaks [4].

Strains belonging to the EPEC group have been subdivided into typical and atypical EPEC as these differ from each other in their adherence mechanisms to human epithelial cells [5] and in their evolutionary lineages [6]. Typical EPEC adhere in a localized manner mediated by bundle-forming pili that are encoded by EAF (EPEC adherence factor) type plasmids harboured by these strains $[5,6]$. Atypical EPEC do not carry EAF plasmids and most of these adhere in a localized adherence-like pattern to epithelial cells [5]. Some EPEC strains share similarities with certain EHEC strains in terms of their $\mathrm{O}: \mathrm{H}$ serotypes, virulence genes and other phaenotypical traits $[5,7,8]$

The chromosomally encoded locus of enterocyte effacement (LEE) which is present in both, EPEC and EHEC strains plays a major role in their pathogenesis. The LEE carries genes for the attaching and effacing phenotype promoting bacterial adhesion and the destruction of human intestinal enterocytes $[2,7,9,10]$. Besides LEE encoded genes, a large number of non-LEE effector genes have been found on prophages and on integrative elements in the chromosome of the typical EPEC strains B171-8 (O111:NM) [11] and 2348/69 (O127:H6) [12]. In a homology-based search, all nonLEE effector families, except cif, found in the typical EPEC strains were also present in EHEC O157:H7 Sakai strain $[11,12]$. On the other hand, some strain specific effectors were only present in EHEC O157:H7 (EspK, EspX) and not in the EPEC strains. Moreover, EPEC O111 and O127 strains were different from each other regarding the presence of some effector genes (EspJ, EspM, EspO, EspV, EspW, NleD, OspB and EspR) $[11,12]$.

It has been shown that EHEC O157:H7 has evolved stepwise from an atypical EPEC O55:H7 ancestor strain $[13,14]$. Atypical EPEC and EHEC strains of serotypes O26, O103, O111 and $\mathrm{O} 145$ have been found to be similar in virulence plasmid encoded genes, tir-genotypes, $t c c P$ genes, LEE and non-LEE encoded genes indicating that these are evolutionarily linked to each other [8,15-19]. The classification of these strains into the EPEC or the EHEC group is merely based on the absence or presence of genes encoding Shiga toxins (Stx) 1 and/or 2. In EHEC strains, stx-genes are typically harboured by transmissible lambdoid bacteriophages and the loss of $s t x$-genes has been described to be frequent in the course of human infection with EHEC $[20,21]$. On the other hand, it has been demonstrated that stx-encoding bacteriophages can convert non-toxigenic $\mathrm{O} 157$ and other E. coli strains into EHEC $[22,23]$.
A molecular risk assessment (MRA) concept has been developed to identify virulent EHEC strains on the basis of non-LEE effector gene typing [24] and a number of nle genes such as nleA, nleB, nleC, nleE, nleF, nleG2, nleG5, nleG6, nleH1-2 and ent/espL2 have been found to be significantly associated with EHEC strains causing HUS and outbreaks in humans $[4,16,17,24]$.

We recently investigated 207 EHEC, STEC, EPEC and apathogenic E. coli strains for the presence of nle genes and EHEC virulence plasmid-associated genes. By statistical analysis, two clusters of strains were obtained. OI122 encoded genes ent/espL2, nleB and $n l e E$ were most characteristic for Cluster 1 , followed by OI-71 encoded genes nleH1-2, nleA and nleF. EHEC-plasmid encoded genes katP, etpD, ehxA, espP, saa and subA showed only medium to low influence on the formation of clusters. Cluster 1 was formed by all EHEC $(\mathrm{n}=44)$ and by eight of twenty-one EPEC strains investigated, whereas Cluster 2 gathered all LEE-negative STEC $(\mathrm{n}=111)$, apathogenic E. coli $(\mathrm{n}=30)$ and the remaining thirteen EPEC strains [17]. These findings indicate that some EPEC strains share non-LEE encoded virulence properties with O157:H7 and other EHEC strains. Such EPEC strains could be derivatives of EHEC which have lost their stxgenes but could also serve as a reservoir for the generation of new EHEC strains by uptake of stx-phages $[16,20,25,26]$.

To classify strains of the EPEC group according to their relationship to EHEC we have investigated 308 typical and atypical EPEC strains for the presence of nle-genes of O-islands OI-57, OI-71 and OI-122, as well as prophage and EHEC-plasmid-associated genes. OI122 encoded genes were found to be significantly associated with atypical EPEC strains that showed close similarities to EHEC regarding their serotypes and other virulence traits. In typical EPEC, the presence of Oisland 122 was significantly associated with strains which are frequently the cause of outbreaks and severe disease in humans.

\section{Results}

Cluster analysis of EHEC, EPEC, STEC and apathogenic E. coli strains

E. coli pathogroups were established as described in the Methods section. The frequencies and associations between virulence genes and E. coli pathogroups are presented in Table 1. The linkage of genes according to their respective PAI or the EHEC-plasmid was $94.7 \%$ (230/243) for OI-122, 41.8\% (142/340) for OI-71, 46.2\% (80/173) for OI-57 and 1.8\% (4/220) for the EHEC-plasmid. As not all PAIs were found to be genetically conserved we decided to perform the cluster analysis on single genes. The results from the cluster analysis using thirteen virulence genes that were taken as cluster 
Table 1 Frequency and associations between virulence genes and $E$. coli pathogroups

\begin{tabular}{|c|c|c|c|c|c|c|}
\hline Genetic element & Virulence gene & $\begin{array}{l}\text { EHEC }(n=64) \\
n, \%(95 \%-C I)^{a}\end{array}$ & $\begin{array}{l}\text { typical EPEC }(n=73) \\
n, \%(95 \%-C l)^{a}\end{array}$ & $\begin{array}{l}\text { atypical EPEC }(n=235) \\
n, \%(95 \%-C I)^{a}\end{array}$ & $\begin{array}{l}\text { STEC }(n=52) \\
n, \%(95 \%-C I)^{a}\end{array}$ & $\begin{array}{l}\text { E. coli }(\mathrm{n}=21) \\
\mathrm{n}, \%(95 \%-\mathrm{Cl})^{\mathrm{a}}\end{array}$ \\
\hline pMAR2 [12] & $b f p A$ & $0,0(0 ; 5.6)$ & $68^{b}, 93.2^{c}(84.7 ; 97.7)$ & $0,0(0 ; 1.6)$ & $0,0(0 ; 6.8)$ & $0,0(0 ; 16.1)$ \\
\hline p0157 [46] & ehxA & $61,95.3^{c}(86.9 ; 99.0)$ & $0,0(0 ; 4.9)$ & $65,27.7(22.0 ; 33.9)$ & $26,50.0^{c}(35.8 ; 64.2)$ & $0,0(0 ; 16.1$ \\
\hline p0157 [46] & espP & $37,57.8^{\mathrm{c}}(44.8 ; 70.1)$ & $1,1.4(0.03 ; 7.4)$ & $26,11.1(7.4 ; 15.8)$ & $14,26.9^{c}(15.6 ; 41.0)$ & $0,0(0 ; 16.1)$ \\
\hline p0157 [46] & etpD & $19,29.7^{c}(18.9 ; 42.4)$ & $3,4.1(0.86 ; 11.5)$ & $79,33.6^{c}(27.6 ; 40.0)$ & $0,0(0 ; 6.8)$ & $0,0(0 ; 16.1)$ \\
\hline p0157 [46] & katP & $36,56.3^{c}(43.3 ; 68.6)$ & $1,1.4(0.03 ; 7.4)$ & $40,17(12.4 ; 22.4)$ & $1,1.9(0.05 ; 10.3)$ & $0,0(0 ; 16.1)$ \\
\hline Ol-71 [31] & nleA & $47,73.4^{c}(60.9 ; 83.7)$ & $17,23.3(14.2 ; 34.6)$ & $119,50.6^{c}(44.1 ; 57.2)$ & $0,0(0 ; 6.8)$ & $0,0(0 ; 16.1)$ \\
\hline Ol-71 [31] & nleF & $45,70.3^{c}(57.6 ; 81.1)$ & $19,26(16.5 ; 37.6$ & $87,37(30.8 ; 43.5)$ & $0,0(0 ; 6.8$ & $0,0(0 ; 16.1)$ \\
\hline Ol-71 [31] & nleH1-2 & $63,98.4^{c}(91.6 ; 100.0)$ & $60,82.2(71.5 ; 90.2)$ & $205,87.2^{c}(82.3 ; 91.2)$ & $0,0(0 ; 6.8)$ & $0,0(0 ; 16.1)$ \\
\hline Ol-122 [31] & ent/espL2 & $64,100.0^{c}(94.4 ; 100.0)$ & $46,63^{\mathrm{C}}(50.9 ; 74.0)$ & $129,54.9(48.3 ; 61.4)$ & $0,0(0 ; 6.8)$ & $0,0(0 ; 16.1)$ \\
\hline Ol-122 [31] & nleB & $64,100.0^{c}(94.4 ; 100.0)$ & $46,63^{\mathrm{C}}(50.9 ; 74.0)$ & $129,54.9(48.3 ; 61.4)$ & $0,0(0 ; 6.8)$ & $0,0(0 ; 16.1$ \\
\hline Ol-122 [31] & nleE & $59,92.2^{\complement}(82.7 ; 97.4)$ & $46,63^{\mathrm{C}}(50.9 ; 74.0)$ & $128,54.5(47.9 ; 61.0)$ & $0,0(0 ; 6.8)$ & $0,0(0 ; 16.1)$ \\
\hline $\mathrm{Ol}-57$ [31] & nleG5 & $33,51.6^{\mathrm{C}}(38.7 ; 64.2)$ & $9,12.3(5.8 ; 22.1)$ & $38,16.2(11.7 ; 21.5)$ & $0,0(0 ; 6.8)$ & $0,0(0 ; 16.1)$ \\
\hline $\mathrm{Ol-57}[31]$ & nleG6-2 & $57,89.1^{c}(78.7 ; 95.5)$ & $9,12.3(5.8 ; 22.1)$ & $107,45.5^{c}(39.0 ; 52.1)$ & $0,0(0 ; 6.8)$ & $0,0(0 ; 16.1)$ \\
\hline CP-933N [31] & espK & $59,92.2^{c}(82.7 ; 97.4)$ & $14,19.2(10.9 ; 30.1)$ & $68,28.9(23.2 ; 35.2)$ & $0,0(0 ; 6.8)$ & $0,0(0 ; 16.1)$ \\
\hline Stx-phage [47] & $s t x_{1}$ & $39,60.9^{c}(47.9 ; 72.9)$ & $0,0(0 ; 4.9)$ & $0,0(0 ; 1.6)$ & $18,34.6^{c}(22.0 ; 49.1)$ & $0,0(0 ; 16.1)$ \\
\hline Stx-phage [31] & $s t x_{2}$ & $33,51.6^{c}(38.7 ; 64.2)$ & $0,0(0 ; 4.9)$ & $0,0(0 ; 1.6)$ & $48,92.3^{c}(81.5 ; 97.9)$ & $0,0(0 ; 16.1)$ \\
\hline LEE [31] & eae & $64,100.0^{c}(94.4 ; 100.0)$ & $73,100^{c}(95.1 ; 100.0)$ & $235,100^{c}(98.4 ; 100.0)$ & $0,0(0 ; 6.8)$ & $0,0(0 ; 16.1)$ \\
\hline
\end{tabular}

a) absolute ( $\mathrm{n}$ ) and relative frequencies (\%) are shown and the exact $95 \%$ confidence level (95\%-CI) [48]; b) five strains have lost the EAF plasmid encoding bfpA upon subculture; c) standardized residuals $>1$ indicates a major influence on a significant chi-square test.

variables are presented in Table 2 . The 445 strains belonging to 151 different serotypes divided into two clusters. Cluster 1 encompassed all 64 EHEC strains, as well as $46(63 \%)$ of the typical and $129(54.9 \%)$ of the atypical EPEC strains. The remaining 133 EPEC strains, as well as all STEC $(\mathrm{n}=52)$ and apathogenic E. coli $(\mathrm{n}$ $=21$ ) were grouped into Cluster 2. The distribution of PAIs and the EHEC-plasmid according to E. coli pathogroups is presented in Figure 1.

The influence of the different virulence genes on the formation of the "EHEC related" Cluster 1 was calculated using the similarity measure of "Rogers and Tanimoto" [27]. The results are presented in Table 3. The OI-122 encoded genes nleB, ent/espL2 and nleE were highly characteristic of Cluster 1 strains (similarity measure > = 0.947). The OI-71 encoded genes nleH1-2, nleA and nleF, as well as nleG6-2 (OI-57) and espK (CP-933N) were also found to be characteristic of Cluster 1 strains but to a lesser degree (similarity measure 0.511-0.684). The presence of the EHEC-plasmid pO157 associated genes and of nleG5-2 (OI-57) had a minor effect on the formation of Cluster 1 (similarity measure $0.382-0.445$ ).

\section{Characteristics of typical EPEC belonging to Clusters 1 and 2}

Forty-six (63\%) of the 73 typical EPEC strains belonging to nine different serotypes were grouped into Cluster 1. Cluster 2 comprised 27 strains belonging to 12 serotypes (Table 2). Typical EPEC Cluster 1 strains were all positive for OI-122 encoded genes ent/espL2, nleB and nleE (similarity measure 1.0), as well as for nleH1-2 (OI-71) (similarity measure 0.678) (Table 4). These genes were absent in typical EPEC Cluster 2 strains, except for nleH1-2 (23.3\% positive). All other genes that were investigated showed only low similarity $(<0.5)$ to Cluster 1 (Table 4).

Table 2 Summary of cluster analysis with strains belonging to different $E$. coli pathogroups

\begin{tabular}{lllllll}
\hline & & \multicolumn{2}{c}{ Cluster $\mathbf{1}$} & \multicolumn{2}{c}{ Cluster 2 } & \multicolumn{2}{c}{ Total } \\
\hline Pathogroup & Strains (\%) & Serotypes (\%) & Strains (\%) & Serotypes (\%) & Strains & Serotypes \\
\hline EHEC & $64(100.0)$ & $14(100)$ & $0(0)$ & 0 & 64 & 14 \\
typical EPEC & $46(63.0)$ & $9(47.4)$ & $27(37.0)$ & $12(63.2)$ & 73 & $19^{\text {a }}$ \\
atypical EPEC & $129(54.9)$ & $40(50.0)$ & $106(45.1)$ & $45(56.25)$ & 235 & $80^{b}$ \\
STEC & $0(0)$ & 0 & $52(100.0)$ & $20(100)$ & 52 & 20 \\
apathogenic E. colic & $0(0)$ & 0 & $21(100.0)$ & $18(100)$ & 21 & 18 \\
all groups & 239 & 63 & 206 & 95 & 445 & 151 \\
\hline
\end{tabular}

a) two serotypes grouped into Clusters 1 and 2 .

b) 5 serotypes grouped each in Cluster 1 and Cluster 2 .

c) faecal isolates from healthy humans or animals which tested negative for eae, bfpA and stx-genes. 


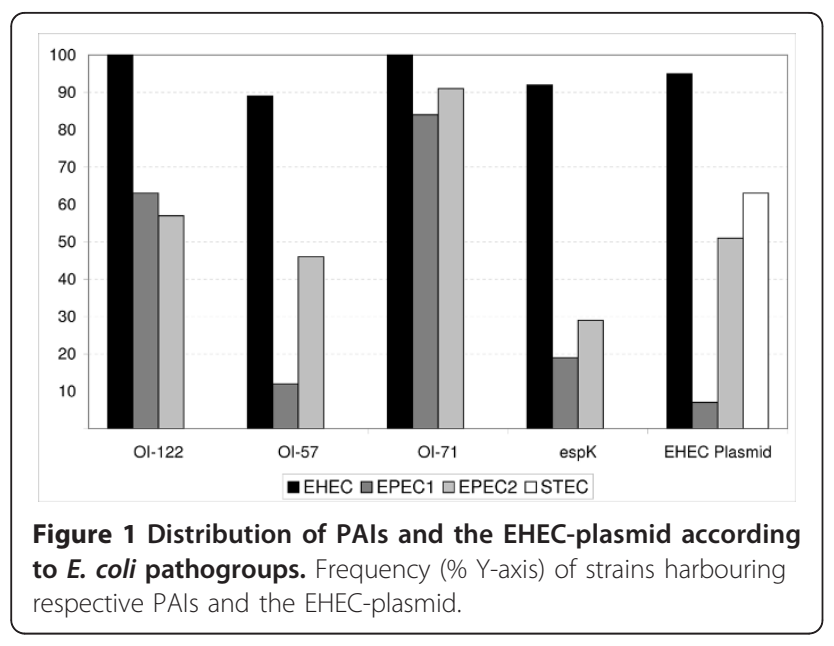

The 73 typical EPEC strains encompassed nineteen different serotypes and one strain was O-rough (Tables 5 and 6). A serotype-specific association with Clusters 1 and 2 was observed. Except for EPEC O119:H6, strains belonging to classical EPEC serotypes such as O55:H6, O111:H2, O114:H2 and O127:H6 grouped in Cluster 1 (Table 5), whereas more rarely observed serotypes were predominant among Cluster 2 strains (Table 6). The single $\mathrm{O} 111: \mathrm{H} 2$ and the $\mathrm{O} 126: \mathrm{H} 27$ strain assigned to Cluster 2 were both negative for all OI-122 associated genes. All other 17 serotypes of typical EPEC were associated with only one cluster each.

\section{Characteristics of atypical EPEC belonging to Clusters 1 and 2}

A total of 235 atypical EPEC strains were investigated (Table 2). Of these, 129 (54.9\%) grouped into Cluster 1. The presence of OI-122 associated genes had the most

Table 3 Similarity measure between virulence genes and Cluster $1 \mathrm{E}$. coli strains from all groups

\begin{tabular}{llc}
\hline Genetic element $^{\mathbf{a}}$ & Virulence gene & Similarity measure \\
\hline Ol-122 & nleB & 1.000 \\
Ol-122 & ent/espL2 & 0.991 \\
Ol-122 & nleE & 0.947 \\
Ol-71 & nleH1-2 & 0.684 \\
Ol-71 & nleF & 0.621 \\
Ol-71 & nleA & 0.553 \\
Ol-57 & nleG6-2 & 0.527 \\
CP-933N & espK & 0.511 \\
pO157 & ehxA & 0.445 \\
Ol-57 & nleG5-2 & 0.440 \\
pO157 & etpD & 0.402 \\
pO157 & espP & 0.399 \\
pO157 & katP & 0.382
\end{tabular}

a) harbouring the virulence gene; b) A value of 1 indicates complete similarity, while a value of zero means no similarity [49].
Table 4 Similarity measure between virulence genes and Cluster 1 for typical EPEC strains

\begin{tabular}{llc}
\hline Genetic element $^{\mathbf{a}}$ & Virulence gene & Similarity measure $^{\mathbf{b}}$ \\
\hline Ol-122 & ent/espL2 & 1.000 \\
Ol-122 & nleB & 1.000 \\
Ol-122 & nleE & 1.000 \\
OI-71 & nleH1-2 & 0.678 \\
Ol-71 & nleA & 0.352 \\
Ol-71 & nleF & 0.352 \\
Ol-57 & nleG5-2 & 0.327 \\
Ol-57 & nleG6-2 & 0.327 \\
CP-933N & espK & 0.315 \\
pO157 & etpD & 0.259 \\
pO157 & espP & 0.237 \\
PO157 & ehxA & 0.227 \\
PO157 & katP & 0.217 \\
\hline
\end{tabular}

a) harbouring the virulence gene; b) A value of 1 indicates complete similarity, while a value of zero means no similarity [49].

influence on the formation of atypical EPEC Cluster 1 strains (similarity measures 0.942-1.0, Table 7). By contrast, only four (3.8\%) of the 106 atypical EPEC of Cluster 2 were positive for OI-122 genes ent/espL2 (one O125: H6 strain) and nleE (one Ont:H52, O157:H39 and O168: H33 strain) and none of the strains was positive for nleB.

The OI-71 encoded genes had only medium influence (similarity measures $0.492-0.649$ ) on the formation of Cluster 1 and OI-57 and EHEC-plasmid encoded genes were of low influence (similarity measures $<0.5)$. Interestingly, EHEC-plasmid genes ehxA ( $\mathrm{p}<0.001), \operatorname{etpD}(\mathrm{p}<$ $0.001), \operatorname{espP}(\mathrm{p}<0.05)$ and $k a t P(\mathrm{p}<0.01)$ were significantly more frequent in atypical EPEC (51.5\% positive) than in typical EPEC (6.9\%) strains (data not shown).

\section{Table 5 Serotypes of typical EPEC Cluster 1 strains}

\begin{tabular}{lcc}
\hline Serotype $^{\mathbf{a}}$ & No. strains & $\%$ \\
\hline O55:H6 & 5 & 10.9 \\
$\mathrm{O} 66: \mathrm{H} 8$ & 1 & 2.2 \\
$\mathrm{O} 111:[\mathrm{H} 2]$ & 17 & 37.0 \\
$\mathrm{O} 111: \mathrm{H} 25$ & 2 & 4.3 \\
$\mathrm{O} 114: \mathrm{H} 2^{\mathrm{b}}$ & 11 & 23.9 \\
$\mathrm{O} 119: \mathrm{H} 2^{\mathrm{c}}$ & 4 & 8.7 \\
$\mathrm{O} 126: \mathrm{H} 27$ & 1 & 2.2 \\
$\mathrm{O} 127: \mathrm{H6}$ & 1 & 2.2 \\
$\mathrm{O} 142:[\mathrm{H} 6]$ & 3 & 6.5 \\
Orough:[H8] & 1 & 2.2 \\
total & 46 & 100.0 \\
\hline
\end{tabular}

a) All strains were isolated from human faeces. In bold: serotypes previously associated with Stx-production [3]. H-type in [brackets] indicates presence of non-motile strains that were investigated for their flic genotype [44]. b) two $0114: \mathrm{H} 2$ strains were positive for the EHEC virulence plasmid associated etpD gene.

c) EPEC O119:H2 were previously reported as atypical EPEC reacting with bfpA probe [5]. One each of the O119:H2 strains was positive for EHEC virulence plasmid associated genes espP and etpD. 
Table 6 Serotypes of typical EPEC Cluster 2 strains

\begin{tabular}{lcc}
\hline Serotype $^{\text {a }}$ & No. strains & $\%$ \\
\hline O55:[H51] & 1 & 3.7 \\
O86:H8 & 5 & 18.5 \\
O86:[H34] & 4 & 14.8 \\
O111:H2 & 1 & 3.7 \\
O111:[H9] & 3 & 11.1 \\
O118:H5 & 1 & 3.7 \\
O119:[H6] & 4 & 14.8 \\
O119:[H52] & 1 & 3.7 \\
O126:H27 & 1 & 3.7 \\
O142:H34 & 1 & 3.7 \\
O157:[H45] & 4 & 14.8 \\
O186:[H45] & 1 & 3.7 \\
Total & 27 & 100.0 \\
\hline
\end{tabular}

a) All strains were from human faeces, except the 0186:H45 strain which was from faeces of a domestic cat. In bold: serotypes previously associated with Stx-production [3]. H-type in [brackets] indicates presence of non-motile strains that were were investigated for their fliC genotype [44].

b) this strain was positive for the EHEC virulence associated katP gene.

The 235 atypical EPEC strains were divided into 80 different serotypes (Table 2). Twenty-five (10.6\%) strains were not typable according to their $\mathrm{O}$-antigens (Tables 8 and 9). With the exception of five serotypes (O26: $\mathrm{H} 11, \mathrm{O} 55: \mathrm{H} 7, \mathrm{O} 103: \mathrm{H} 2, \mathrm{O} 128: \mathrm{H} 2$ and $\mathrm{O} 145: \mathrm{H} 28)$, all others were associated with one cluster only. Strains belonging to classical EHEC types O26:H11, O103:H2, $\mathrm{O} 145: \mathrm{H} 28$ and $\mathrm{O} 157: \mathrm{H} 7(\mathrm{n}=30)$ were predominant in Cluster 1 (23.3\%) (Table 8). Only four strains (3.8\%) of classical EHEC serotypes grouped into Cluster 2 (Table 9). These were one avian O26:H11 strain (negative for OI-122 and OI-71 genes), one human O103:H2 and two human O145:H28 strains (all negative for OI-122 and OI-71 genes except for nleH1-2).

Table 7 Similarity measure between virulence genes and Cluster 1 for atypical EPEC strains

\begin{tabular}{llc}
\hline Genetic element $^{\mathbf{a}}$ & Virulence factor & Similarity measure $^{\mathbf{b}}$ \\
\hline Ol-122 & nleB & 1.000 \\
Ol-122 & ent/espL2 & 0.983 \\
Ol-122 & nleE & 0.942 \\
Ol-71 & nleF & 0.649 \\
Ol-71 & nleA & 0.511 \\
Ol-71 & nleH1-2 & 0.492 \\
Ol-57 & nleG6-2 & 0.429 \\
pO157 & ehxA & 0.420 \\
CP-933N & espK & 0.399 \\
pO157 & etpD & 0.395 \\
pO157 & espP & 0.382 \\
Ol-57 & nleG5-2 & 0.382 \\
pO157 & katP & 0.313 \\
\hline
\end{tabular}

a) Harbouring the virulence gene; b) A value of 1 indicates complete similarity, while a value of zero means no similarity [49].
Table 8 Serotypes of atypical EPEC Cluster 1 strains

\begin{tabular}{|c|c|c|c|}
\hline Serotype $^{a}$ & No. strains & Origin $^{b}$ & $\%$ \\
\hline $\mathrm{O} 2:[\mathrm{H} 40]$ & 3 & $h(1), a(2)$ & 2.3 \\
\hline O3:[H8] & 3 & h (3) & 2.3 \\
\hline 015:[H2] & 2 & h (2) & 1.6 \\
\hline O26:[H11 $]^{c}$ & 20 & h (9), a (11) & 15.5 \\
\hline O55:H7 & 17 & h (17) & 13.2 \\
\hline $\mathrm{O} 70: \mathrm{H} 11^{\mathrm{d}}$ & 5 & a (5) & 3.9 \\
\hline O76:[H7] $]^{\mathrm{e}}$ & 5 & h (5) & 3.9 \\
\hline $\mathrm{O} 80:[\mathrm{H} 2]^{\mathrm{d}}$ & 3 & a (3) & 2.3 \\
\hline O86:H11 & 2 & h (2) & 1.6 \\
\hline O100:[H25] & 2 & $h(1)^{d}, a(1)$ & 1.6 \\
\hline O103:H2 ${ }^{d}$ & 2 & a (2) & 1.6 \\
\hline $0103: \mathrm{H}^{2} 5^{\mathrm{d}}$ & 4 & $a(3), f(1)$ & 3.1 \\
\hline 0111:[H11] $]^{d}$ & 2 & h (2) & 1.6 \\
\hline O117:[H40] & 3 & h (3) & 2.3 \\
\hline O118:[H8] & 3 & h (3) & 2.3 \\
\hline O119:[H8] & 2 & $h(1), a(1)^{d}$ & 1.6 \\
\hline $0119:[\mathrm{H} 25]^{\mathrm{d}}$ & 3 & h (2), a (1) & 2.3 \\
\hline O127:[H40] & 7 & h (7) & 5.4 \\
\hline O128:[H2] & 3 & h (3) & 2.3 \\
\hline $0145:[\mathrm{H} 28]^{\mathrm{d}}$ & 5 & $h(4), a(1)$ & 3.9 \\
\hline O156:H8 & 2 & $h(1), a(1)^{d}$ & 1.6 \\
\hline 0157:[H7] ${ }^{d}$ & 3 & h (3) & 2.3 \\
\hline $0177: H 11^{d}$ & 2 & a (2) & 1.6 \\
\hline Ont:[H2] ${ }^{\mathrm{d}}$ & 2 & h (2) & 1.6 \\
\hline Ont:[H21] & 4 & h (4) & 3.1 \\
\hline Orough:[H40] & 2 & h (2) & 1.6 \\
\hline single ${ }^{f}$ & 18 & see footnote to table & 14.0 \\
\hline total & 129 & & 100.0 \\
\hline
\end{tabular}

a) in bold: serotypes previously associated with Stx-production [3].

b) Origin and numbers (in brackets) of strains $h=$ human, $a=$ animal, $f=$ food.

c) Four O26:H11 strains from humans and seven from animals were positive for the EHEC-plasmid associated ehxA gene.

d) All strains were positive for $e h x A$.

e) One strain was positive for $e h x A$.

f) serotypes and strains represented each by one isolate only: $\mathrm{O} 2 \mathrm{H} 8$ (a), O3: H5 (f), O3:H40 (h), O15:H11 (a), O21:H25 (h), O22:[H7] (h), O45:H7 (a), O71:H40 (h), O76:H41 (a),O84:[H2] (a), O109:H25 (a), O117:H25 (h), O121:H- (h), O121: H19 (h), O127:H8 (h), O128:H8 (h), O153:H14 (a), and Orough:[H7] (h).

Forty (50\%) of the 80 serotypes encompassing atypical EPEC were associated with strains carrying one or more of the EHEC-plasmid genes ehxA, katP, etpD, espP. EHECplasmid genes $\operatorname{etp} D(\mathrm{p}<0.01), e h x A(\mathrm{p}<0.001)$ and espP $(\mathrm{p}<0.001)$ were significantly more frequent among strains $(89 / 129=69 \%)$ and serotypes $(28 / 40=70 \%)$ belonging to Cluster 1 than in strains $(32 / 106=30.2 \%)$ and serotypes $(15 / 46=32.6 \%)$ of Cluster 2 (data not shown).

\section{Presence of virulence genes in STEC and apathogenic $E$. coli strains}

The 52 STEC strains investigated in this study belonged to 20 different serotypes (Table 2). Twelve of these (O113:H4, O113:H21, O118:H12, O146:H28, O153:H25, 
Table 9 Serotypes of atypical EPEC Cluster 2 strains

\begin{tabular}{|c|c|c|c|}
\hline Serotype $^{a}$ & No. strains & Origin $^{b}$ & $\%$ \\
\hline$\overline{\mathrm{O} 28:[\mathrm{H} 28]^{\mathrm{C}}}$ & 4 & h (4) & 3.8 \\
\hline O49:H10 & 3 & $h(1), a(2)^{c}$ & 2.8 \\
\hline O51:H49 & 3 & h (3) & 2.8 \\
\hline O55:H7 & 2 & h (2) & 1.9 \\
\hline O63:H6 & 2 & h (2) & 1.9 \\
\hline O69:H16 & 2 & a (2) & 1.9 \\
\hline O108:H9 ${ }^{d}$ & 6 & $a(6)$ & 5.7 \\
\hline O111:H19 & 3 & h (3) & 2.8 \\
\hline O113:H6 & 2 & h (2) & 1.9 \\
\hline O114:[H49] & 5 & h (5) & 4.7 \\
\hline O115:[H38] & 3 & h (3) & 2.8 \\
\hline O123:H45 & 2 & h (2) & 1.9 \\
\hline O125:H6 & 3 & h (3) & 2.8 \\
\hline 0128:[H2] & 10 & h (9), a (1) & 9.4 \\
\hline $0145:[H 28]^{d}$ & 2 & h (2) & 1.9 \\
\hline O145:[H34] & 5 & h (5) & 4.7 \\
\hline O157:[H16] & 4 & $h(3), f(1)$ & 3.8 \\
\hline O157:H26 & 2 & h (2) & 1.9 \\
\hline Ont:[H2] & 3 & $h(1), a(2)$ & 2.8 \\
\hline Ont:H6 & 2 & a (2) & 1.9 \\
\hline single $e^{e}$ & 38 & see footnote to table & 35,8 \\
\hline Total & 106 & & 100.0 \\
\hline
\end{tabular}

a)in bold: serotypes previously associated with Stx-production [3].

b)Origin and numbers (in brackets) of strains $h=$ human, $a=$ animal, $f=$ food c)One strain was positive for the ehxA gene

d)All strains were positive for $e h x A$

e)serotypes and strains represented each by one isolate only: O4:H16 (a), O5: $\mathrm{H}-(\mathrm{a}), \mathrm{O} 5: \mathrm{H}_{11} 1^{\mathrm{d}}$ (a), O8:[H10] (h), O9:H10 (h), O26:[H11] (a), O37:H10 (a), O45:H9 (h), O62:H9 ${ }^{\mathrm{d}}(\mathrm{a}), \mathrm{O} 65:[\mathrm{H} 25](\mathrm{h}), \mathrm{O} 69:[\mathrm{H} 2)(\mathrm{h}), \mathrm{O} 0: \mathrm{H} 2(\mathrm{~h}), \mathrm{O} 88: \mathrm{H} 8$ (h), O102:[H19] (h), O103:H2 ${ }^{\text {d }}$ (h), O119:H9 (h), O123:[H25] (h), O127:[H19] (h), O127:H21 (h), $\mathrm{O} 145: \mathrm{H}^{\mathrm{d}}$ (a), O145:H19 (a), O150:H8 (f), O157:H2 (a), O157:H39 (h), O168: [H33] (h), O177:H26 (h), O177:H6 (a), Ont:H7 ${ }^{\mathrm{d}}$ (a), Ont:H10 (h), Ont:H11 (a), Ont: H14 (h), Ont:[H24] (h), Ont:[H26] (h), Ont:H40 (h), Ont:[H52] (h), Orough:H6 (h), Orough:H9 (h), and Orough:H10 (h).

O174:H8, O22:H8, O22:H16, O76:H19, O8:H19, O91: $\mathrm{H} 10$ and $\mathrm{O} 91: \mathrm{H} 21)$ were previously described from isolates of human origin [3]. Apart from stx-genes, 33 (63.5\%) of 52 STEC were positive for one or more of EHEC-plasmid associated genes $\operatorname{ehxA}$, espP and katP. None of the STEC was positive for the plasmid etpD gene as for all other nle-genes investigated in this study (Table 1). The 21 apathogenic E. coli strains belonged to 18 different serotypes (Table 2) and were negative for all virulence markers investigated in this study (Table 1).

\section{Discussion}

The concept of molecular risk assessment [24] has been successfully employed for grouping STEC strains into those that are associated with outbreaks and life-threatening disease in humans and those which cause less severe or are not implicated in human disease. The presence of non-LEE effector genes encoded by $\mathrm{O}$-islands OI-122, OI-71 and OI-57 has been shown to be highly associated with EHEC strains that were frequently involved in outbreaks and severe disease in humans $[4,16,17,24,28,29]$. In a previous work, we were able to associate the presence of OI-122 and OI-71 encoded genes with an "EHEC-Cluster" comprising forty-four EHEC strains as well as eight of twenty-one EPEC strains investigated [17]. This finding indicates that some EPEC strains are more related to EHEC in their virulence patterns, than others.

In order to explore this relationship between EPEC and EHEC more closely, we investigated larger numbers of strains and serotypes of typical and atypical EPEC for thirteen virulence genes associated with EHEC O157 Oislands OI-122, OI-71, OI-57, the EHEC-plasmid and prophage CP-933N. Genes for nleG5-2 and nleG6-2 were included since OI-57 specific genes were previously found to be associated with classical EHEC and also with some EPEC strains [24,28]. The prophage CP933 associated espK gene was included since its homologues were found in EHEC O157, O26, O103 and O111, in atypical EPEC O55:H7 but not in typical EPEC O127 and $\mathrm{O} 111$ strains $[11,12,14,30,31]$.

Our findings indicate that about half of the typical and atypical EPEC strains and serotypes are closely related to EHEC regarding these virulence attributes (Table 2). The presence of OI-122 encoded genes, followed by OI71 were most significant for the assignment of EPEC to the "EHEC-related" Cluster 1 confirming data from our previous study performed on a different collection of strains [17]. The OI-57 encoded genes nleG5-2 and nleG6-2, as well as the espK gene were not as strongly associated with Cluster 1, as the OI-122 and OI-71 genes. Recently, the OI-57 associated genes adfO and $c k f$ were reported to be present in 30 (71\%) of 42 investigated EPEC strains but a high variability of OI-57 associated orfs in EPEC strains was observed [28]. This could explain the results of our study, where the OI-57 associated nleG5-2 gene was found infrequently in all EPEC, whereas the nleG6-2 gene was frequent in atypical EPEC (45.5\%) but rarely found in typical EPEC (12.3\%) (Table 1). Further work is needed to define the genes of OI-57 that are most suitable for the molecular risk assessment of EHEC and EPEC strains.

In our study, EHEC-plasmids were associated with EHEC, STEC and atypical EPEC, but not with typical EPEC strains. EHEC-plasmids are frequently harboured by classical EHEC but also by many LEE-negative STEC strains [32-34]. Correspondingly, EHEC-plasmid encoded genes $\operatorname{eh} x A$, etpD, katP and espP had only a small influence on Cluster 1 formation, confirming results of previous studies [16,17]. In this study, EHECplasmid genes were significantly more associated with atypical EPEC Cluster 1 than with Cluster 2 strains. The high proportion of EHEC-plasmid positives among 
Cluster 1 strains suggests that many of these may have derived from EHEC by losing stx-genes. A loss of stxgenes was reported to occur frequently in classical EHEC strains $[23,26]$. EHEC-plasmid genes were found in $23 / 29(79.3 \%)$ of atypical EPEC Cluster 1 strains belonging to EHEC related serotypes O26:H11, O103: H2, O145:H28 and O157:H7 (data not shown). These 30 EHEC-like strains showed the same virulence characteristics (presence of OI-122 genes) as their homologous EHEC strains.

In addition to this, there are epidemiological findings pointing to a closer relationship between "Cluster 1 " atypical EPEC and EHEC strains. Significantly $(p<0.05)$ more typable $(78 / 120=65.0 \%)$ Cluster 1 strains than Cluster 2 strains belonged to serotypes $(18 / 40=45.0 \%)$ that are associated with the production of Shiga toxins (38). Only 26.6\% (24/90) of the atypical EPEC strains of Cluster 2 showed O:H types $(10 / 46=21.7)$ previously associated with Stx-production.

Typical EPEC were also found to split into Cluster 1 and Cluster 2 strains. Cluster 1 was formed by typical EPEC serotypes O55:H6, O114:H2, O111:[H2], O127:H6 and O142:H6 strains which accounted worldwide for large outbreaks in hospitals, infant wards and day nurseries with a high mortality rate [35-37]. Cluster 2 typical EPEC accounted for serotypes that were more rarely associated with outbreaks, except for EPEC O119:H6, the latter was frequently associated with infantile diarrhoea in Brazil $[38,39]$. On the basis of these findings, a seropathotype classification for typical EPEC similar to those described for STEC [4,24] can be established. Typical EPEC strains associated with outbreaks and high mortality are gathered in Cluster 1 which is mainly characterized by the presence of OI-122 associated genes ent/espL2, nleB, nleE. These findings are supported by two clinical studies showing that the presence of OI-122 encoded genes was significantly associated with diarrhoea in patients infected with atypical EPEC $[40,41]$. The function of nle-genes in pathogenesis of EHEC and EPEC infection is only partially known $[30,42,43]$. Further work is needed to explore the contribution of OI-122 effectors to the high infectivity and virulence of EPEC and EHEC strains resulting in outbreaks and severe disease in humans.

It has been shown previously that the evolution of typical and atypical EPEC has occurred from LEE positive ancestor strains and divergent phylogenetic groups of EPEC (EPEC1 to EPEC4) and EHEC (EHEC1 and EHEC2) were established [1,6,37]. Virulence genes harboured by EAF-plasmids, EHEC-plasmids and stx-phages were found in phylogenetically unrelated strains indicating that these were acquired several times during evolution [1]. Their horizontal spread to unrelated strains and the frequent loss of plasmid and bacteriophage inherited determinants makes these less suitable for identifying clones associated with high infectivity and virulence in humans. The OI-122 inherited nle-genes were found to be significantly associated with highly virulent Cluster 1 strains of EHEC and EPEC. They appear to be more stably inherited than plasmid and phage associated genes and could thus serve as an additional diagnostic tool for the reliable identification of EHEC and EPEC infections in humans, animals and EHEC contamination of food sources and the environment.

\section{Conclusion}

Our results indicate that the OI-122 pathogenicity island is a common attribute that is significantly associated with highly virulent EHEC and EPEC strains. Of the OI122 encoded genes, nleB was found as most conserved and thus presents a suitable marker for genetic screening for human virulent EHEC and EPEC strains. Horizontally transferred genetic elements such as the virulence-plasmids and phages were less significantly associated with the highly virulent clones of EHEC and EPEC strains.

\section{Methods \\ Bacteria}

A total of $445 E$. coli strains from the collection of the National Reference Laboratory for Escherichia coli (NRL-E.coli) were investigated. These originated from humans $(\mathrm{n}=286)$, domestic animals $(\mathrm{n}=84)$ and food $(\mathrm{n}=70)$. Five strains were of unknown origin. The 445 strains were grouped into apathogenic E. coli $(\mathrm{n}=21)$, atypical EPEC ( $\mathrm{n}=235)$, typical EPEC $(\mathrm{n}=73)$, EHEC $(\mathrm{n}=64)$ and STEC $(\mathrm{n}=52)$ according to the presence or absence of genes encoding Stx $\left(s t x_{1}+s t x_{2}\right)$, intimin (eae) and bundle forming pili $(b f p A)$. All strains were investigated for their $\mathrm{O}$ (lipopolysaccharide) and $\mathrm{H}$ (flagellar) serotypes. Non-motile strains were examined for their flagellar $(f l i C)$ genotype as previously described [44]. Highly purified total DNA of the strains was prepared from $0.5 \mathrm{ml}$ overnight cultures of bacteria using the RTP $^{\circledR}$ Bacteria DNA Mini Kit (Invitek, Berlin, Germany).

\section{Detection of genes by real-time PCR}

To investigate the presence of seventeen genes previously described as virulence markers of STEC, EPEC and EHEC the real-time PCR method was employed using the GeneDisc ${ }^{\circledR}$ array as previously described [17], or the Applied Biosystems 7500 real time PCR system. Standard cycling conditions $\left(15 \mathrm{sec} 94^{\circ} \mathrm{C}, 1 \mathrm{~min} 60^{\circ} \mathrm{C}\right.$ and 40 cycles) were used for the Applied Biosystems 7500 system. The primers and probes for the detection of following genes $\left(s t x_{1}, s t x_{2}\right.$, eae, ehxA, espP etpD, katP, nleA, nleF, nleH1-2 ent/espL2, nleB, nleE) have been 
described previously [16]. Primers and probes for the detection of $b f p A$, nleG5-2, nleG6-2 and espK were developed for this work (Table 10). The reference strains for STEC and EHEC were used as previously described [16]. Strain E2348/69 (O127:H6) [12] served as control for typical EPEC and strain CB9615 (O55:H7) [14] as a control of atypical EPEC. E. coli $\mathrm{K}-12$ strain MG1655 [45] served as a negative control for the eighteen virulence markers investigated in this work.

\section{Definition of E. coli pathogroups}

The genes eae, stx $x_{1} s t x_{2}$ and $b f p A$ were used to define $E$. coli pathogroups and were therefore not taken as independent variables for the cluster/statistical analysis. On the genotype basis, the strains were grouped as atypical EPEC (eae only), typical EPEC (eae and $b f p A$ ), STEC $\left(s t x_{1}\right.$ and/or $\left.s t x_{2}\right)$, EHEC (eae and $s t x_{1}$ and/or $\left.s t x_{2}\right)$ and apathogenic $E$. coli (absence of eae, bfpA, stx $x_{1}$ and $s t x_{2}$ ). The presence of the following genes was used to define clusters of EHEC related and EHEC unrelated strains as previously described [17]: ehxA, espP, etpD and katP (EHEC-virulence plasmid pO157), nleA, nleF and nleH12 (OI-71), ent/espL2, nleB and nleE (OI-122). These variables proved to be useful for the characterization of STEC and EHEC strains $[4,16,17,24,29]$. In addition to this, genes nleG5-2 and nleG6-2 (OI-57) [24] and espK (prophage CP-933N) [31] had previously been found to be associated with EHEC [11,12,24,25,28] and therefore included as new variables for the cluster analysis.

\section{Statistical analysis}

The seventeen virulence genes that were investigated in the 445 E. coli strains are listed in Table 1. To analyse the relationship between the seventeen virulence factors investigated in this work and the E. coli pathogroups, the presence of the virulence factors was calculated per pathogroup (Table 1). For the analysis of associations between the virulence factors and the $E$. coli pathogroups univariate analysis with a chi-square test was used. If frequencies were low Fisher's exact tests was used for the calculation. As a significance level, $\alpha$ was set to 0.05 . All $\mathrm{p}$-values $\leq \alpha$ were considered statistically significant. To determine which virulence genes were major contributors in the elimination of the null hypothesis we calculated standardized residuals. When the absolute value of the residual is greater than 1.00 we can conclude that there is a major influence on a significant chi-square test between a given pathotype and the respective virulence gene (Table 1). A cluster analysis was performed in order to analyse similarities between the E. coli pathogroups. Since the presence or absence of virulence genes is binary scaled, the similarity was calculated according to "Rogers and Tanimoto" [27]. The linkage between groups was selected as the cluster method.

\section{Acknowledgements}

The work is part of the thesis of MB, a PhD student financially supported by ANSES. Part of the work was carried out at the NRL-E.coli in Berlin under the supervision of LB. The authors are grateful to Katja Steege, Sabine Haby and Karin Pries for their technical assistance.

\section{Author details}

${ }^{1}$ ANSES (French Agency for Food, Environmental and Occupational Health Safety), Food Safety Laboratory, 23 Av du Général De Gaulle, Fr-94706 Maisons-Alfort, France. ${ }^{2}$ Epidemiology, Biostatistics and Mathematical Modelling, Scientific Services, Federal Institute for Risk Assessment (BfR), Diedersdorfer Weg 1 D-12277 Berlin, Germany. ${ }^{3}$ National Reference Laboratory for Escherichia coli (NRL-E.coli), Federal Institute for Risk Assessment (BfR), Diedersdorfer Weg 1 D-12277 Berlin, Germany.

\section{Authors' contributions}

$L B$ and PF played an integral role in the project conception and MB, PF and $\mathrm{LB}$ in method development. MB was mainly responsible for the design and execution of the experimental procedures. Data processing and statistical analysis was done by AM. Data analysis and interpretation of the results was completed by all authors. LB was mostly responsible for the preparation of the manuscript. All authors have read and approved the final manuscript.

\section{Competing interests}

The authors declare that they have no competing interests.

Table 10 Primers and probes for real-time PCR detection of virulence genes developed for this study

\begin{tabular}{|c|c|c|c|}
\hline Target gene $^{a}$ & Forward primer, reverse primer and probe sequences $\left(5^{\prime}-3^{\prime}\right)$ & Location within sequences & Gene Bank accession no. \\
\hline \multirow[t]{3}{*}{ nleG6-2 (Z2150) } & ATATGCTCTCTATATGATAAGGATG & $1928877-1928901$ & AE005174 \\
\hline & AAAGTGACATTCGTCTITTCTCATA & 1928996-1928872 & \\
\hline & [6FAM]CGTTAGTGCAACTTGTTGAAACTGGTGGAA[BHQ1] & $1928902-1928931$ & \\
\hline \multirow[t]{3}{*}{ nleG5-2 (Z2151) } & AGACTATTCGTGGAGAAGCTCAAG & 1929199-1929222 & AE005174 \\
\hline & TATTGAAGGCCAATCTGGATG & 1929337-1929317 & \\
\hline & [6FAM]TGGATATITATGGGAAGTCTTAACCAGGATGG[BHQ1] & 1929269-1929301 & \\
\hline \multirow[t]{3}{*}{ espk } & ATTGTAACTGATGTTATTTCGTTTGG & $1673295-1673320$ & AE005174 \\
\hline & GRCATCAAAAGCGAAATCACACC & $1673419-1673397$ & \\
\hline & [6FAM]CAGATACTCAATATCACAATCTTTGATATATAAACGACC[BHQ1] & 1673330-1673368 & \\
\hline \multirow[t]{3}{*}{ bfpA } & CCAGTCTGCGTCTGATTCCA & $2756-2775$ & FM180569 \\
\hline & CGTTGCGCTCATTACTTCTGAA & 2816-2795 & \\
\hline & TAAGTCGCAGAATGC-MGB & $2777-2791$ & \\
\hline
\end{tabular}

a) Z2150 and Z2151 derive from OI-57 [24]. 
Received: 16 March 2011 Accepted: 21 June 2011

Published: 21 June 2011

\section{References}

1. Donnenberg MS, Whittam TS: Pathogenesis and evolution of virulence in enteropathogenic and enterohemorrhagic Escherichia coli. J Clin Invest 2001, 107:539-548

2. Robins-Browne RM, Hartland EL: Escherichia coli as a cause of diarrhea. $J$ Gastroenterol Hepatol 2002, 17:467-475.

3. Scheutz F, Strockbine NA: Genus I. Escherichia. In Bergey's Manual of Systematic Bacteriology.. 2 edition. Edited by: Garrity GM, Brenner DJ, Krieg NR, Staley JT. Springer; 2005:607-624.

4. Karmali MA, Mascarenhas M, Shen S, Ziebell K, Johnson S, Reid-Smith R, et al: Association of Genomic O Island 122 of Escherichia coli EDL 933 with Verocytotoxin-Producing Escherichia coli Seropathotypes That Are Linked to Epidemic and/or Serious Disease. J Clin Microbiol 2003, 41:4930-4940.

5. Trabulsi LR, Keller R, Tardelli Gomes TA: Typical and atypical enteropathogenic Escherichia coli. Emerg Infect Dis 2002, 8:508-513.

6. Lacher DW, Steinsland H, Blank TE, Donnenberg MS, Whittam TS: Molecular evolution of typical enteropathogenic Escherichia coli: clonal analysis by multilocus sequence typing and virulence gene allelic profiling. $J$ Bacteriol 2007, 189:342-350

7. Campos LC, Franzolin MR, Trabulsi LR: Diarrheagenic Escherichia coli categories among the traditional enteropathogenic E. coli O serogroups-a review. Mem Inst Oswaldo Cruz 2004, 99:545-552.

8. Kozub-Witkowski E, Krause G, Frankel G, Kramer D, Appel B, Beutin L: Serotypes and virutypes of enteropathogenic and enterohaemorrhagic Escherichia coli strains from stool samples of children with diarrhoea in Germany. J Appl Microbiol 2008, 104:403-410.

9. Campellone KG: Cytoskeleton-modulating effectors of enteropathogenic and enterohaemorrhagic Escherichia coli: Tir, EspFU and actin pedestal assembly. FEBS J 2010, 277:2390-2402.

10. Clarke SC, Haigh RD, Freestone PPE, Williams PH: Virulence of Enteropathogenic Escherichia coli, a Global Pathogen. Clin Microbiol Rev 2003, 16:365-378.

11. Ogura $Y$, Abe H, Katsura K, Kurokawa $K$, Asadulghani M, lguchi $A$, et al: Systematic identification and sequence analysis of the genomic islands of the enteropathogenic Escherichia coli strain B171-8 by the combined use of whole-genome PCR scanning and fosmid mapping. J Bacteriol 2008, 190:6948-6960

12. Iguchi A, Thomson NR, Ogura Y, Saunders D, Ooka T, Henderson IR, et al: Complete genome sequence and comparative genome analysis of enteropathogenic Escherichia coli 0127:H6 strain E2348/69. J Bacteriol 2009, 191:347-354

13. Wick LM, Qi W, Lacher DW, Whittam TS: Evolution of genomic content in the stepwise emergence of Escherichia coli 0157:H7. J Bacteriol 2005, 187:1783-1791

14. Zhou Z, Li X, Liu B, Beutin L, Xu J, Ren Y, et al: Derivation of Escherichia coli 0157:H7 from its 055:H7 precursor. PLoS One 2010, 5:e8700.

15. Abu-Ali GS, Lacher DW, Wick LM, Qi W, Whittam TS: Genomic diversity of pathogenic Escherichia coli of the EHEC 2 clonal complex. BMC Genomics 2009, 10:296

16. Bugarel $M$, Beutin $L$, Fach P: Low-density macroarray targeting non-locus of enterocyte effacement effectors (nle genes) and major virulence factors of Shiga toxin-producing Escherichia coli (STEC): a new approach for molecular risk assessment of STEC isolates. Appl Environ Microbiol 2010, 76:203-211.

17. Bugarel M, Beutin L, Martin A, Gill A, Fach P: Micro-array for the identification of Shiga toxin-producing Escherichia coli (STEC) seropathotypes associated with Hemorrhagic Colitis and Hemolytic Uremic Syndrome in humans. Int J Food Microbiol 2010, 142:318-329.

18. Miko A, Lindstedt BA, Brandal LT, Lobersli I, Beutin L: Evaluation of multiple-locus variable number of tandem-repeats analysis (MLVA) as a method for identification of clonal groups among enteropathogenic, enterohaemorrhagic and avirulent Escherichia coli 026 strains. FEMS Microbiol Lett 2010, 303:137-146.

19. Bielaszewska M, Zhang W, Tarr PI, Sonntag AK, Karch H: Molecular profiling and phenotype analysis of Escherichia coli O26:H11 and O26:NM: secular and geographic consistency of enterohemorrhagic and enteropathogenic isolates. J Clin Microbiol 2005, 43:4225-4228.
20. Bielaszewska M, Middendorf B, Kock R, Friedrich AW, Fruth A, Karch $H$, et al: Shiga toxin-negative attaching and effacing Escherichia coli: distinct clinical associations with bacterial phylogeny and virulence traits and inferred in-host pathogen evolution. Clin Infect Dis 2008, 47:208-217.

21. Bielaszewska M, Kock R, Friedrich AW, von Eiff C, Zimmerhackl LB, Karch $H$, et al: Shiga toxin-mediated hemolytic uremic syndrome: time to change the diagnostic paradigm? PLOS ONE 2007, 2:e1024.

22. Dopfer D, Sekse C, Beutin L, Solheim H, van der Wal FJ, de BA, et al Pathogenic potential and horizontal gene transfer in ovine gastrointestinal Escherichia coli. J Appl Microbiol 2010, 108:1552-1562.

23. Mellmann A, Lu S, Karch H, Xu Jg, Harmsen D, Schmidt MA, et al: Recycling of Shiga Toxin 2 Genes in Sorbitol-Fermenting Enterohemorrhagic Escherichia coli 0157:NM. Appl Environ Microbiol 2008, 74:67-72.

24. Coombes BK, Wickham ME, Mascarenhas M, Gruenheid S, Finlay BB, Karmali MA: Molecular analysis as an aid to assess the public health risk of non-0157 Shiga toxin-producing Escherichia coli strains. Appl Environ Microbiol 2008, 74:2153-2160.

25. Bugarel M, Beutin L, Scheutz F, Loukiadis E, Fach P: Identification of genetic markers for differentiation of Shiga toxin-producing, enteropathogenic and avirulent strains of Escherichia coli 026. Appl Environ Microbiol 2011, 77:2275-2281.

26. Bielaszewska M, Prager R, Kock R, Mellmann A, Zhang W, Tschape H, et al: Shiga Toxin Gene Loss and Transfer In Vitro and In Vivo during Enterohemorrhagic Escherichia coli O26 Infection in Humans. Appl Environ Microbiol 2007, 73:3144-3150.

27. Zuur AF, leno EN, Smith GM: Measures of association. In Analysing Ecological Data. Edited by: Gaij M, Krickeberg K, Samet J, Tsiatis A, Wong W. New York: Springer; 2007:163-187.

28. Imamovic L, Tozzoli R, Michelacci V, Minelli F, Marziano ML, Caprioli A, et al: Ol-57, a genomic island of Escherichia coli 0157, is present in other seropathotypes of Shiga toxin-producing E. coli associated with severe human disease. Infect Immun 2010, 78:4697-4704.

29. Konczy P, Ziebell K, Mascarenhas M, Choi A, Michaud C, Kropinski AM, et al: Genomic $O$ island 122, locus for enterocyte effacement, and the evolution of virulent verocytotoxin-producing Escherichia coli. J Bacterio 2008, 190:5832-5840.

30. Ogura Y, Ooka T, Iguchi A, Toh H, Asadulghani M, Oshima K, et al: Comparative genomics reveal the mechanism of the parallel evolution of 0157 and non-0157 enterohemorrhagic Escherichia coli. Proc Natl Acad Sci USA 2009, 106:17939-17944.

31. Perna NT, Plunkett G III, Burland V, Mau B, Glasner JD, Rose DJ, et al: Genome sequence of enterohaemorrhagic Escherichia coli 0157:H7 Nature 2001, 409:529-533.

32. Boerlin P, Chen S, Colbourne JK, Johnson R, De GS, Gyles C: Evolution of enterohemorrhagic Escherichia coli hemolysin plasmids and the locus for enterocyte effacement in shiga toxin-producing $E$. coli. Infect Immun 1998, 66:2553-2561.

33. Brunder W, Schmidt H, Frosch M, Karch H: The large plasmids of Shigatoxin-producing Escherichia coli (STEC) are highly variable genetic elements. Microbiology 1999, 145(Pt 5):1005-1014.

34. Newton HJ, Sloan J, Bulach DM, Seemann T, Allison CC, Tauschek M, et al: Shiga toxin-producing Escherichia coli strains negative for locus of enterocyte effacement. Emerg Infect Dis 2009, 15:372-380.

35. Beutin L. Orskov I, Orskov F, Zimmermann S, Prada J, Gelderblom H, et al: Clonal diversity and virulence factors in strains of Escherichia coli of the classic enteropathogenic serogroup 0114. J Infect Dis 1990, 162:1329-1334.

36. Edelman R, Levine MM: From the National Institute of Allergy and Infectious Diseases. Summary of a workshop on enteropathogenic Escherichia coli. J Infect Dis 1983, 147:1108-1118.

37. Whittam TS, McGraw EA: Clonal analysis of EPEC serogroups. Revista de Microbiologia 1996, 27:7-16.

38. Toledo MR, Alvariza MC, Murahovschi J, Ramos SR, Trabulsi LR: Enteropathogenic Escherichia coli serotypes and endemic diarrhea in infants. Infect Immun 1983, 39:586-589.

39. Gomes TA, Vieira MA, Wachsmuth IK, Blake PA, Trabulsi LR: Serotypespecific prevalence of Escherichia coli strains with EPEC adherence factor genes in infants with and without diarrhea in Sao Paulo, Brazil. J Infect Dis 1989, 160:131-135.

40. Vieira MA, Salvador FA, Silva RM, Irino K, Vaz TM, Rockstroh AC, et al: Prevalence and characteristics of the 0122 pathogenicity island in 
typical and atypical enteropathogenic Escherichia coli strains. J Clin Microbiol 2010, 48:1452-1455.

41. Afset JE, Bruant G, Brousseau R, Harel J, Anderssen E, Bevanger L, et al: Identification of virulence genes linked with diarrhea due to atypical enteropathogenic Escherichia coli by DNA microarray analysis and PCR. J Clin Microbiol 2006, 44:3703-3711.

42. Dean P, Kenny B: The effector repertoire of enteropathogenic E. coli: ganging up on the host cell. Curr Opin Microbiol 2009, 12:101-109.

43. Spears KJ, Roe AJ, Gally DL: A comparison of enteropathogenic and enterohaemorrhagic Escherichia coli pathogenesis. FEMS Microbiol Lett 2006, 255:187-202

44. Beutin L, Miko A, Krause G, Pries K, Haby S, Steege K, et al: Identification of human-pathogenic strains of Shiga toxin-producing Escherichia coli from food by a combination of serotyping and molecular typing of Shiga toxin genes. Appl Environ Microbiol 2007, 73:4769-4775.

45. Riley M, Abe T, Arnaud MB, Berlyn MK, Blattner FR, Chaudhuri RR, et al: Escherichia coli K-12:a cooperatively developed annotation snapshot2005. Nucleic Acids Res 2006, 34:1-9.

46. Burland V, Shao Y, Perna NT, Plunkett G, Sofia HJ, Blattner FR: The complete DNA sequence and analysis of the large virulence plasmid of Escherichia coli 0157:H7. Nucl Acids Res 1998, 26:4196-4204.

47. Calderwood SB, Auclair F, Donohue-Rolfe A, Keusch GT, Mekalanos JJ: Nucleotide sequence of the Shiga-like toxin genes of Escherichia coli. Proc Natl Acad Sci USA 1987, 84:4364-4368.

48. Collett D: Modelling binary data Boca Raton, Florida: Chapman \& Hall/CRC; 1999

49. Bühl A: SPSS Version 16: Einführung in die moderne Datenanalyse. 11 edition. Munich: Pearson Studium; 2008.

doi:10.1186/1471-2180-11-142

Cite this article as: Bugarel et al: Virulence gene profiling of enterohemorrhagic (EHEC) and enteropathogenic (EPEC) Escherichia coli strains: a basis for molecular risk assessment of typical and atypical EPEC strains. BMC Microbiology 2011 11:142.

\section{Submit your next manuscript to BioMed Central and take full advantage of:}

- Convenient online submission

- Thorough peer review

- No space constraints or color figure charges

- Immediate publication on acceptance

- Inclusion in PubMed, CAS, Scopus and Google Scholar

- Research which is freely available for redistribution

Submit your manuscript at www.biomedcentral.com/submit
Biomed Central 